\title{
One-shot BRDF imaging system to obtain surface properties
}

\author{
Hiroshi Ohno ${ }^{1}$ (D) Takahiro Kamikawa ${ }^{1}$
}

Received: 21 March 2021 / Accepted: 23 July 2021 / Published online: 6 August 2021

(c) The Author(s) 2021

\begin{abstract}
The bidirectional reflectance distribution function (BRDF) that describes an angle-resolved distribution of surface reflectance is available for characterizing surface properties of a material. A one-shot BRDF imaging system can capture an in-plane color mapping of light direction extracted from a surface BRDF distribution. A surface roughness identification method is then proposed here using the imaging system. A difference between surface properties of a matt paper and a glossy paper is experimentally shown to be detected using the method. A surface reconstruction method of an axisymmetric micro-object using the imaging system is also proposed here. The imaging system experimentally shows that it can reconstruct an axisymmetric aluminium cone surface with a height of $37 \mu \mathrm{m}$.
\end{abstract}

\section{Introduction}

In many manufacturing processes, material surfaces are inspected for quality control by means of several optical systems. For example, a surface roughness identification or a defect detection is needed for quality control. It is, however, often difficult to capture a small surface roughness or a micro-defect by a conventional camera. A measurement of a surface shape of a micro-object is also often needed. In metal bonding processes such as welding [1], soldering, and brazing, a contact angle between a solid plate and a molten metal is an indicator of adhesion, and is needed to be measured for quality check. There are several methods to measure the contact angle with images captured by cameras $[2,3]$. When the contact angle is too small, however, it is difficult to measure the contact angle accurately. Moreover, it is often difficult to obtain an in-plane distribution of the surface shape of the micro-object.

The bidirectional reflectance distribution function (BRDF) that describes an angle-resolved distribution of surface reflectance is available for characterizing physical properties of a material surface $[4,5]$. Indeed, surface

Hiroshi Ohno

hiroshi1.ohno@toshiba.co.jp

Corporate Research \& Development Center, Toshiba

Corporation, Komukai Toshiba-cho 1, Saiwai-ku,

Kawasaki 212-8582, Japan roughness and surface normal vector affect the BRDF. An imaging system that can obtain color mapping of light ray direction extracted from a surface BRDF distribution has recently been developed [6-9]. The imaging system is here called a one-shot BRDF imaging system or briefly one-shot BRDF. Methods to obtain surface properties such as the surface roughness distribution and surface normal vector distribution using the one-shot BRDF are then proposed here.

In this work, two methods to obtain surface properties are proposed. One method is an identification method of surface roughness using the one-shot BRDF imaging system. The other method is a reconstruction method of a three-dimensional axisymmetric surface. The remainder of this paper is organized as follows. First, a basic structure of the one-shot BRDF imaging system is described. Second, an identification method of surface roughness using the one-shot BRDF is proposed, which is experimentally validated using a matt paper and a glossy paper. Third, a three-dimensional surface reconstruction method of an axisymmetric micro-object is also proposed, which is experimentally validated using an aluminium cone with a height of $37 \mu \mathrm{m}$ and a FWHM (full width at half maximum) of about $1000 \mu \mathrm{m}$. The reconstructed three-dimensional surface agrees with that measured by means of the scanning white light interferometer microscope (ZYGO) [10]. Lastly, conclusions are described. 


\section{One-shot BRDF imaging system}

Figure 1 shows a schematic cross-sectional view of the one-shot BRDF imaging system. The one-shot BRDF consists mainly of both a parallel light illumination optical system and an imaging optical system. The illumination optical system has an LED and a collimator lens that can convert the diverging light rays emitted from the LED to collimated parallel light rays. The collimated parallel light rays are reflected by a beam splitter and travel toward a material surface. The imaging optical system has an imaging lens and a multicolor filter that consists of concentric regions with different color filters $[11,12]$. The multicolor filter is placed at the focal plane of the imaging lens at a distance $f$ from a principal plane of the imaging lens. The optical axis of the imaging lens is set to $z$-axis in a Cartesian coordinate system of $(x, y, z)$ with its origin

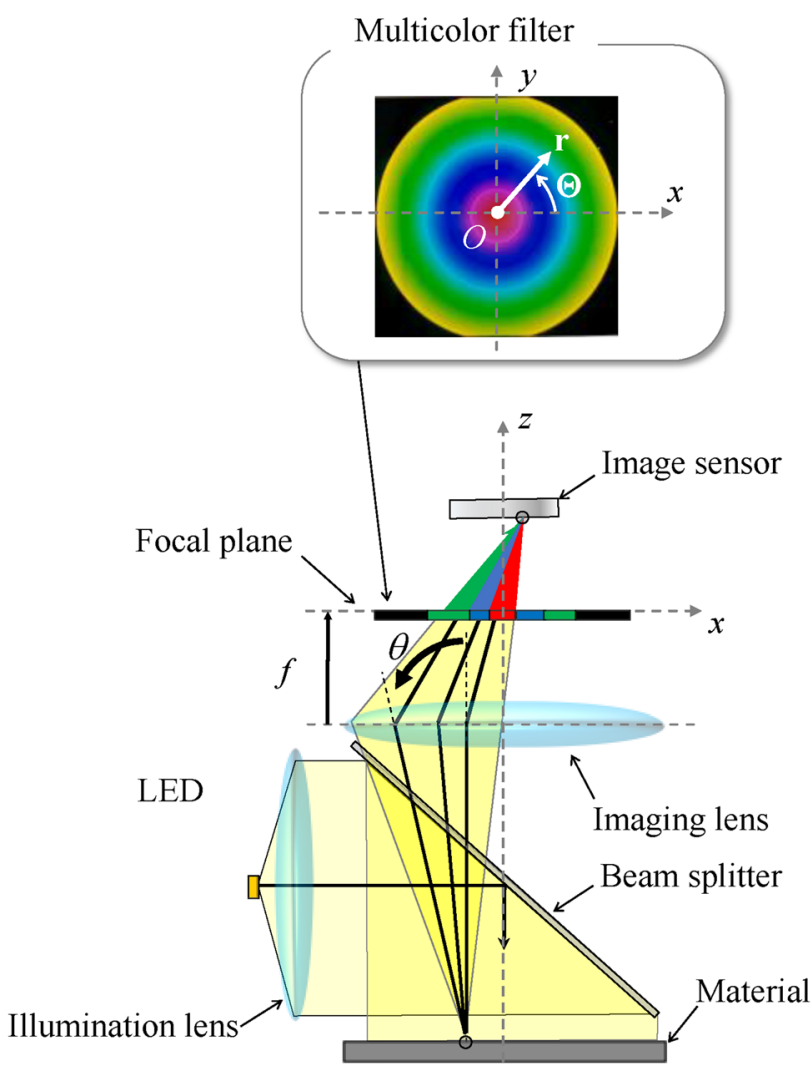

Fig. 1 Schematic cross-sectional view of one-shot BRDF imaging system (one-shot BRDF). The one-shot BRDF consists mainly of a parallel light illumination optical system and an imaging optical system. The imaging optical system has an imaging lens and a multicolor filter that is placed at the focal plane of the imaging lens. The optical axis of the imaging lens is set to $z$-axis in a Cartesian coordinate system of $(x, y, z)$. The multicolor filter is parallel to the $x-y$ plane corresponding to the center of the multicolor filter. The multicolor filter is parallel to the $x-y$ plane.

The reflected light rays from the material surface pass through the beam splitter and will be refracted by the imaging lens. The refracted light rays pass through the multicolor filter and will be imaged on an image sensor. In this way, a light ray reflected by an object point on the material surface is imaged on an imaging point on the image sensor with its color selected depending on its direction.

A light ray with an angle $\theta$ with respect to the optical axis passes through a position $\mathbf{r}$ in the multicolor filter. The radial distance $r$ of the position $\mathbf{r}$ from the optical axis can be derived based on the geometrical optics under the paraxial approximation as

$r=f \theta$.

A two-dimensional angle vector $\boldsymbol{\theta}$ is defined here as

$\boldsymbol{\theta} \equiv\left(\begin{array}{c}\theta_{x} \\ \theta_{y}\end{array}\right) \equiv\left(\begin{array}{c}\theta \cos \Theta \\ \theta \sin \Theta\end{array}\right)=\frac{\mathbf{r}}{f}$,

where the $\theta_{x}$ and $\theta_{y}$ denote the two-dimensional component of the angle vector, and $\Theta$ denotes an azimuth angle with respect to the $x$-axis.

A schematic half-cut view of a prototype of the oneshot BRDF imaging system is shown in Fig. 2. The crosssectional plane includes the optical axis of the one-shot

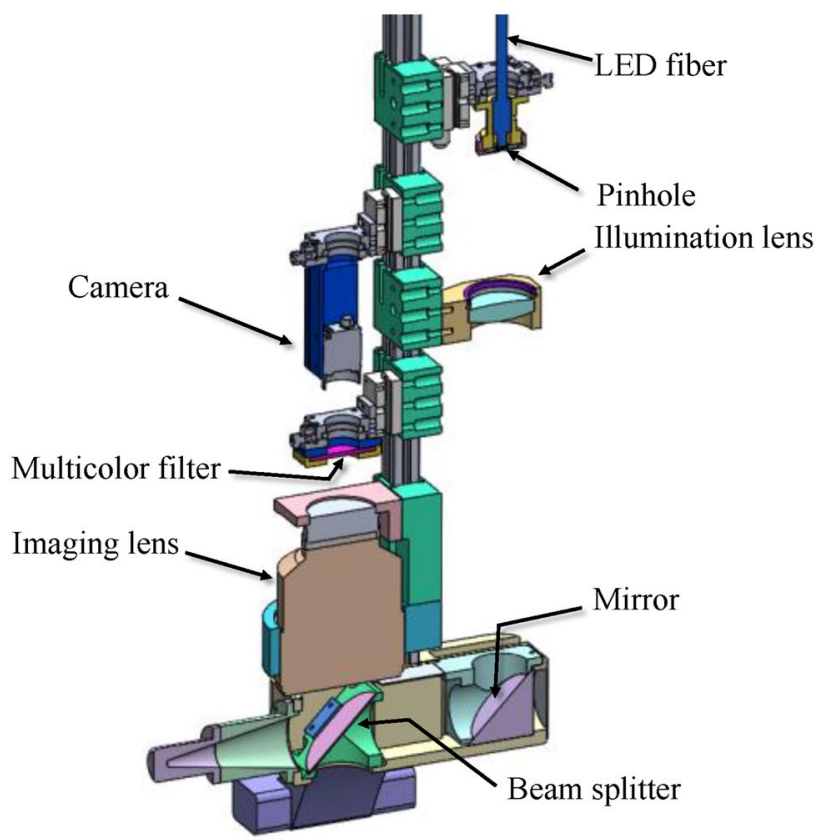

Fig. 2 Schematic half-cut view of a prototype of the one-shot BRDF imaging system. The cross-sectional plane includes the optical axis of the one-shot BRDF. In this work, the focal length $f$ of the imaging lens is set to $105 \mathrm{~mm}$. A focal length of the illumination collimator lens is set to $150 \mathrm{~mm}$ 
BRDF. In this work, the focal length $f$ of the imaging lens (Nikon, AF-S NIKKOR F/1.4) is set to $105 \mathrm{~mm}$. The maximum capturable angle $\theta$ of a light ray with respect to the optical axis is about $7^{\circ}$. A focal length of the illumination collimator lens is set to $150 \mathrm{~mm}$. A diameter of the pinhole is set to $1000 \mu \mathrm{m}$. A divergence angle of the collimated illumination light is thus estimated to be $0.38^{\circ}$. A spatial resolution of an image captured by the one-shot BRDF is determined by both a spatial resolution of the image sensor and a magnification of the optical system.

\section{Surface roughness identification using the one-shot BRDF}

\subsection{Surface roughness identification method}

A surface BRDF is affected by surface roughness of a material. A color component ratio obtained by the image sensor will, therefore, be affected by the surface roughness. In other words, color component ratios of two different surfaces are the same if surface roughnesses of the two surfaces are the same. On the other hand, the color component ratios are different if the two surface roughnesses are different.

A color-position vector $\mathbf{C}$ is here defined using intensity of $I_{m}(m=1,2, \ldots, M)$ of each $m$ th color channel at a pixel position of $\mathbf{Q}=(k, l)$ in the image sensor as

$\mathbf{C}(\mathbf{Q})=I_{1}(\mathbf{Q}) \mathbf{e}_{1}+I_{2}(\mathbf{Q}) \mathbf{e}_{2}+\cdots+I_{M}(\mathbf{Q}) \mathbf{e}_{M}$,

where $\mathbf{e}_{m}(m=1,2, \ldots, M)$ denotes an orthonormal basis defined as

$\mathbf{e}_{1}=\left(\begin{array}{c}1 \\ 0 \\ \vdots \\ 0\end{array}\right), \mathbf{e}_{2}=\left(\begin{array}{c}0 \\ 1 \\ \vdots \\ 0\end{array}\right), \ldots, \mathbf{e}_{M}=\left(\begin{array}{c}0 \\ \vdots \\ 0 \\ 1\end{array}\right)$.

Note that the color-position vector $\mathbf{C}$ varies depending on the multicolor filter.

An angle $\alpha$ between two color-position vectors at different pixel positions, namely $\mathbf{Q}$ and $\mathbf{Q}^{\prime}$, can be written as

$\alpha=\cos ^{-1}\left(\frac{\mathbf{C}(\mathbf{Q}) \cdot \mathbf{C}\left(\mathbf{Q}^{\prime}\right)}{\sqrt{\mathbf{C}(\mathbf{Q}) \cdot \mathbf{C}(\mathbf{Q})} \sqrt{\mathbf{C}\left(\mathbf{Q}^{\prime}\right) \cdot \mathbf{C}\left(\mathbf{Q}^{\prime}\right)}}\right)$.

Note that the angle $\alpha$ becomes 0 if directions of the two color-position vectors at the respective pixel positions of $\mathbf{Q}$ and $\mathbf{Q}^{\prime}$ are the same. On the other hand, the angle $\alpha$ becomes more than 0 if the directions of the color-position vectors are different. The surface roughness can, therefore, be identified by the angle $\alpha$.

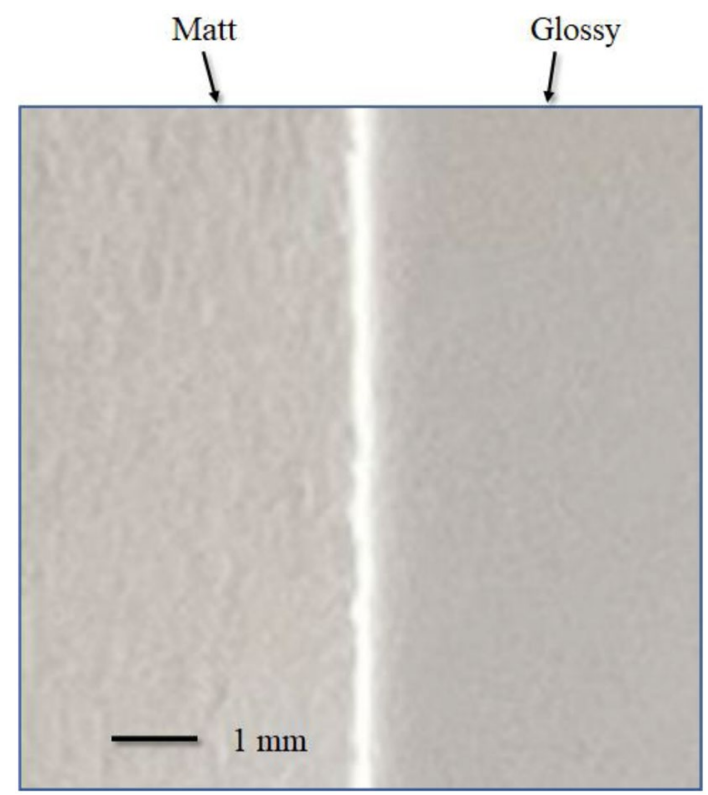

Fig. 3 Image of two papers captured by a conventional camera under an ordinary ambient lighting. A paper shown on the left-hand side is matt paper. The other paper shown on the right-hand side is glossy paper

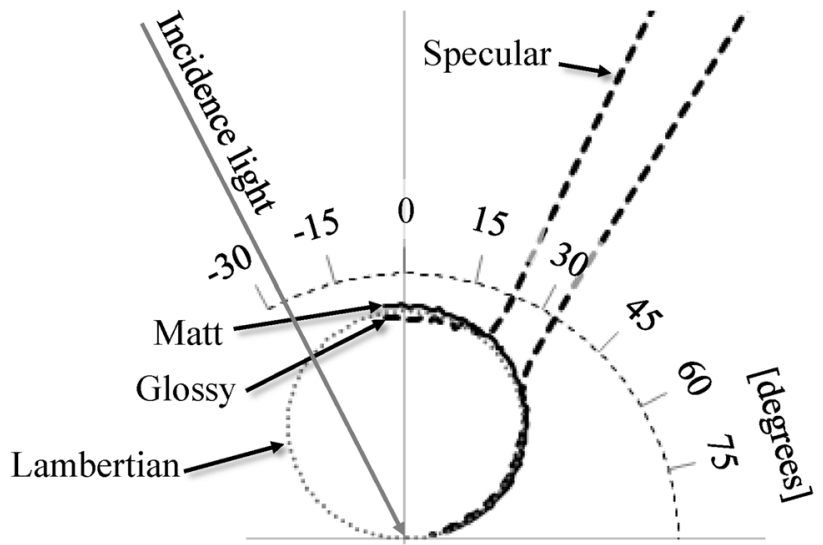

Fig. 4 Goniometric BRDF measurements of matt and glossy papers with an incident light angle of $-28^{\circ}$. Solid black line denotes the BRDF of the matt paper, and dashed black line denotes that of the glossy paper. An ideal Lambertian reflection is also plotted with dotted line

\subsection{Experimental validation}

Figure 3 shows an image of two papers captured by a conventional camera under an ordinary ambient lighting. A paper shown on the left-hand side is matt paper (Kokuyo, $\mathrm{KJ}$-2635). The other paper shown on the right-hand side is glossy paper (Kokuyo, LBP-FG3635).

Figure 4 shows goniometric BRDF measurements for the matt and glossy papers with an incident collimated light 
that has an incident angle of $-28^{\circ}$. Solid black line denotes the BRDF of the matt paper, and dashed black line denotes that of the glossy paper. An ideal Lambertian reflection is also plotted with dotted line. The BRDF of the matt paper approximates the Lambertian reflection whereas that of the glossy paper has a specular reflection.

Figure 5 shows a multicolor filter used for the experiment. The multicolor filter has a blue color filter at its center whereas it has a red color filter surrounding the center. The outermost diameter of the multicolor filter is $23 \mathrm{~mm}$.

Figure 6 shows an unprocessed image of two papers captured by the one-shot BRDF imaging system. A paper shown on the left-hand side is matt paper. The other paper shown on the right-hand side is glossy paper.

A two-dimensional color-position vector $\mathbf{C}_{2 \mathrm{D}}$ is here defined using intensities of $I_{\mathrm{R}}$ and $I_{\mathrm{B}}$ of the red and blue color channels, respectively, at a pixel position of $\mathbf{Q}=(k$, l) as

$\mathbf{C}_{2 \mathrm{D}}(\mathbf{Q})=I_{\mathrm{R}}(\mathbf{Q}) \mathbf{e}_{1}+I_{\mathrm{B}}(\mathbf{Q}) \mathbf{e}_{2}$.

An angle $\alpha_{2 \mathrm{D}}$ between two color-position vectors at different pixel positions, namely $\mathbf{Q}$ and $\mathbf{Q}^{\prime}$, can be written using Eqs. (5) and (6) as

$\alpha_{2 \mathrm{D}}=\cos ^{-1}\left(\frac{I_{\mathrm{R}}(\mathbf{Q}) I_{\mathrm{R}}\left(\mathbf{Q}^{\prime}\right)+I_{\mathrm{B}}(\mathbf{Q}) I_{\mathrm{B}}\left(\mathbf{Q}^{\prime}\right)}{\sqrt{\mathbf{C}_{2 \mathrm{D}}^{2}(\mathbf{Q})} \sqrt{\mathbf{C}_{2 \mathrm{D}}^{2}\left(\mathbf{Q}^{\prime}\right)}}\right)$.

The angle $\alpha_{2 \mathrm{D}}$ becomes 0 if surface roughnesses at corresponding pixel positions of $\mathbf{Q}$ and $\mathbf{Q}^{\prime}$ are the same whereas it becomes more than 0 if those surface roughnesses are different. The surface roughness can, therefore, be identified by the direction of the color-position vector $\mathbf{C}_{2 \mathrm{D}}$.

Figure 7 shows color-position vectors for the matt and glossy papers. A blue dot indicates an endpoint of the

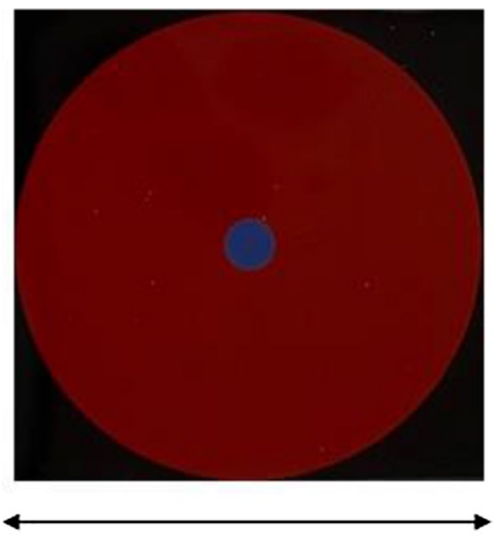

$23 \mathrm{~mm}$

Fig. 5 Multicolor filter used for the experiment

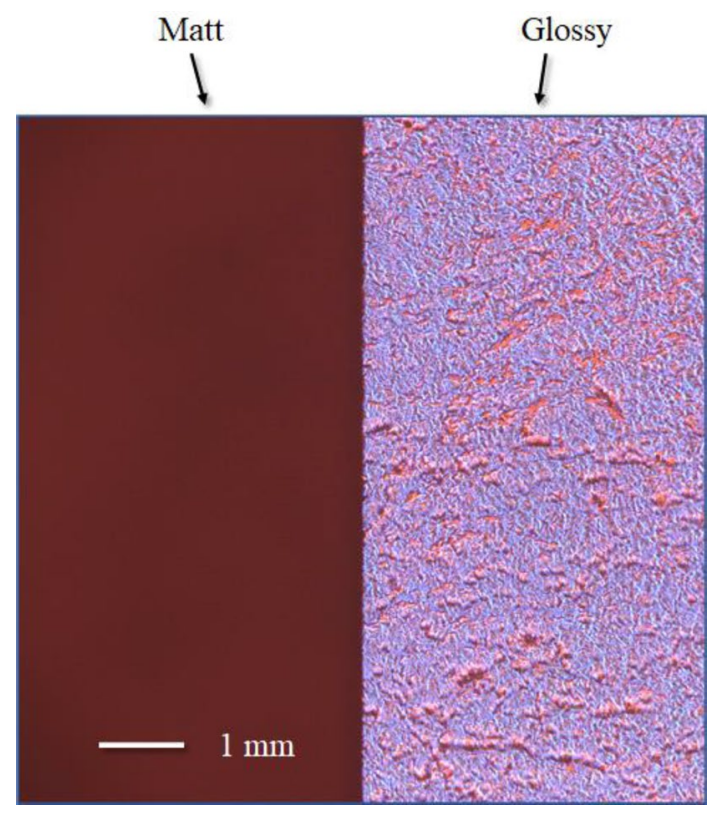

Fig. 6 Unprocessed image of two papers captured by the one-shot BRDF imaging system. A paper shown on the left-hand side is matt paper. The other paper shown on the right-hand side is glossy paper

color-position vector of the glossy paper. A red dot indicates an endpoint of the color-position vector of the matt paper. Two vectors averaged over the two entire areas of the matt and glossy papers are also respectively plotted with bold gray arrow. The averaged color-position vector $\mathbf{C}_{2 \mathrm{D} \text {,matt }}$ is $(42,16)$ whereas that for the glossy paper, $\mathbf{C}_{2 \mathrm{D} \text {,glossy }}$ is

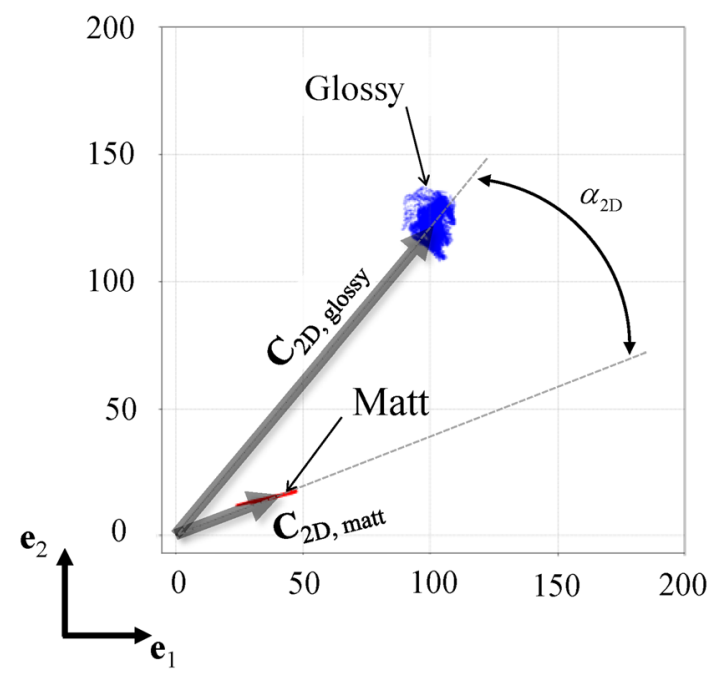

Fig. 7 Endpoints of color-position vectors for the matt and glossy papers. Horizontal axis denotes an axis parallel to $\mathbf{e}_{1}$, and vertical axis denotes an axis parallel to $\mathbf{e}_{2}$. A color-position vector $\mathbf{C}_{2 \mathrm{D} \text {,matt }}$ averaged over the entire area of the matte paper is $(42,16)$ whereas that for the glossy paper, $\mathbf{C}_{2 \mathrm{D} \text {,glossy }}$ is $(101,121)$. The angle $\alpha_{2 \mathrm{D}}$ between the two averaged vectors of $\mathbf{C}_{2 \mathrm{D} \text {, matt }}$ and $\mathbf{C}_{2 \mathrm{D} \text {,glossy }}$ is $29^{\circ}$ 
$(101,121)$. The angle $\alpha_{2 \mathrm{D}}$ between the two averaged vectors of $\mathbf{C}_{2 \mathrm{D} \text {,matt }}$ and $\mathbf{C}_{2 \mathrm{D} \text {,glossy }}$ is $29^{\circ}$, which is calculated using Eq. (7). The difference of the surface roughnesses can, therefore, be quantitatively evaluated using the color-position vectors

\section{Reconstruction of axisymmetric micro-object using the one-shot BRDF}

\subsection{Reconstruction method}

A height of a micro-object is set to $\Psi(x, y)$ as a function of $x$ and $y$, which denotes a distance along the $z$-axis from a reference plane (i.e., $z=z_{0}$ ). The micro-object surface is assumed to be specular. In this case, an angle of an incidence light ray with respect to a surface normal vector is equal to an angle of a reflected light ray with respect to the surface normal vector. An inclination angle of the surface normal vector with respect to the optical axis is assumed to be sufficiently small. The two-dimensional angle vector $\boldsymbol{\theta}$ represented by Eq. (2) can then be approximated [6] as

$\boldsymbol{\theta} \simeq-2\left(\begin{array}{c}\partial_{x} \Psi \\ \partial_{y} \Psi\end{array}\right)=-2 \nabla \Psi(x, y)$.

This equation indicates that the two-dimensional angle vector $\boldsymbol{\theta}$ can be derived from the gradient of the scalar potential, which ensures that the scalar potential can be inversely obtained from the angle vector [13-16].

Assuming that the micro-object is axisymmetric, the light ray angle $\theta$ with respect to the optical axis can be derived using Eq. (8) as

$\theta=2 \sqrt{\left(\partial_{x} \Psi\right)^{2}+\left(\partial_{y} \Psi\right)^{2}}=2\left|\frac{\partial \Psi}{\partial r}\right|$.

The $\Psi$ can, therefore, be derived by integrating of Eq. (9) over the radius $r$ as

$\Psi=\frac{1}{2} \int_{r}^{\infty} d r\left[\frac{1}{2 \pi} \int_{0}^{2 \pi} d \Theta \theta(r, \Theta)\right]$,

where the angle $\theta$ is obtainable by means of the one-shot BRDF using the multicolor filter color mapping [6-8]. Note that the observed angle $\theta$ is written as a function of the $r$ and $\Theta$. In the derivation of Eq. (10) from Eq. (9), the microobject is assumed to be convex instead of concave.

\subsection{Experimental validation}

A micro-object of an aluminium cone is fabricated by a machining process on an aluminium plate. Figure 8 shows (a)

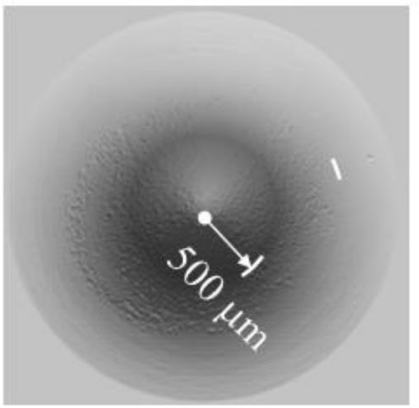

\section{(b)}
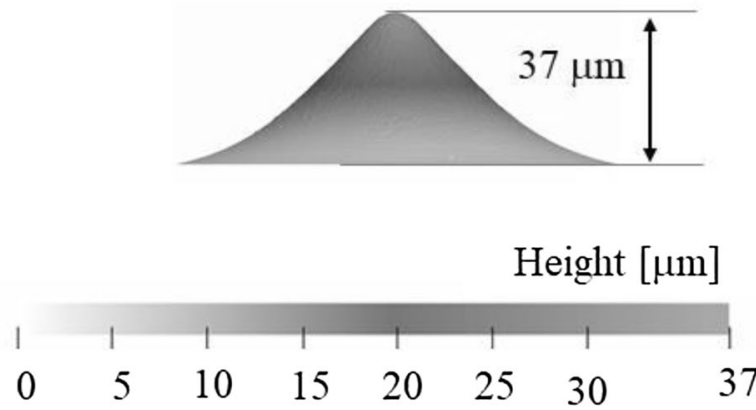

Fig. 8 Three-dimensional surface of an aluminium cone is measured by the scanning white light interferometer microscope (ZYGO). The cone is shown (a) in top view and (b) in side view. Height of the cone is contoured in grayscale

a three-dimensional surface of the aluminium cone that is measured by the scanning white light interferometer microscope (ZYGO). The scanning time is about a few seconds. The cone is shown (a) in top view and (b) in side view. Height of the cone is contoured in grayscale. The cone has a height of $37 \mu \mathrm{m}$ with a cross-sectional FWHM (Full Width at Half Maximum) of about $1000 \mu \mathrm{m}$. The cone surface is well polished, which ensures that the surface roughness is sufficiently small. The apex angle of the cone is about $176^{\circ}$, which means that an inclination angle of the surface normal vector is about $2^{\circ}$.

Figure 9 shows (a) an unprocessed image of an in-plane view of the aluminium cone obtained by means of the oneshot BRDF imaging system. The multicolor filter is set to have 32 graded color regions with outermost diameter of $23 \mathrm{~mm}$ where the red region is center as shown in Fig. 10. In Fig. 9, the image directly reveals an in-plane surface inclination angle distribution. A color contour scale is constructed by one-shot BRDF images of a well-polished aluminium plate with various inclination angles to the optical axis at intervals of $0.25^{\circ}$.

Figure 11 shows a reconstructed surface using Eq. (10) with the captured image shown in Fig. 9. The surface measured by the scanning white light interferometer microscope 

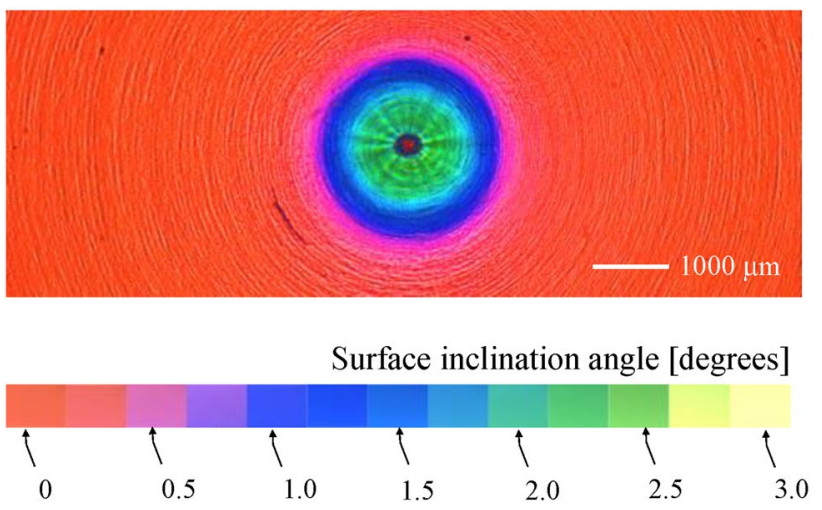

Fig. 9 Unprocessed image of aluminium cone captured by the oneshot BRDF imaging system. A color contour scale is constructed by one-shot BRDF images of a polished aluminium plate with various inclination angles to the optical axis at intervals of $0.25^{\circ}$

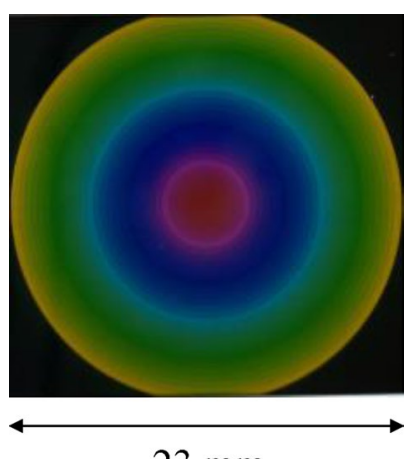

$23 \mathrm{~mm}$

Fig. 10 Multicolor filter used for the experiment
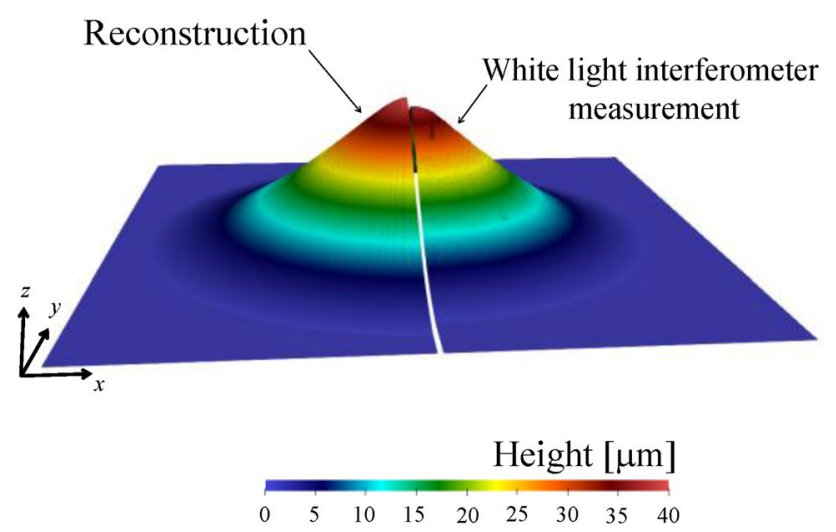

Fig. 11 Reconstructed surface using Eq. (10) with the captured image shown in Fig. 9. The surface measured by the scanning white light interferometer microscope is also plotted. The left half denotes the reconstruction whereas the right half denotes the white light interferometer measurement. Height of the surface is color-contoured is also plotted. Height of the surface is color-contoured. The left half denotes the reconstruction whereas the right half denotes the white light interferometer measurement. The maximum difference of the reconstruction from the white light interferometer measurement is about $3 \mu \mathrm{m}$, which corresponds to about $8 \%$ error. The reconstruction agrees well with the scanning white light interferometer measurement, which validates the axisymmetric reconstruction method.

\section{Conclusions}

The BRDF that describes an angle-resolved distribution of surface reflectance is available for characterizing surface properties of a material. A one-shot BRDF imaging system can capture an in-plane color mapping of light direction extracted from a surface BRDF distribution. Two methods to obtain the surface properties using the one-shot BRDF imaging system are then proposed.

A surface roughness identification method represented by Eq. (5) using the one-shot BRDF imaging system is proposed in the Sect. 3. The difference between surface properties of a matt paper and a glossy paper is experimentally shown to be detected using the method. An angle between two color-position vectors for the matt and glossy papers becomes $29^{\circ}$, which validates that the surface roughness can be identified by the one-shot BRDF.

A reconstruction method of an axisymmetric microobject using the one-shot BRDF imaging system is also proposed, which is represented by Eq. (10) in the Sect. 4. The one-shot BRDF imaging system experimentally shows that it can reconstruct an axisymmetric aluminium cone with a maximum error of about $8 \%$.

\section{Declarations}

Conflict of interest The authors declare no conflicts of interest.

Open Access This article is licensed under a Creative Commons Attribution 4.0 International License, which permits use, sharing, adaptation, distribution and reproduction in any medium or format, as long as you give appropriate credit to the original author(s) and the source, provide a link to the Creative Commons licence, and indicate if changes were made. The images or other third party material in this article are included in the article's Creative Commons licence, unless indicated otherwise in a credit line to the material. If material is not included in the article's Creative Commons licence and your intended use is not permitted by statutory regulation or exceeds the permitted use, you will need to obtain permission directly from the copyright holder. To view a copy of this licence, visit http://creativecommons.org/licenses/by/4.0/. 


\section{References}

1. Ohno, H., Shiomi, Y., Tsuno, S., Sasaki, M.: Design of a transmissive optical system of a laser metal deposition three-dimensional printer with metal powder. Appl. Optics 58(15), 4127-4138 (2019)

2. Dutra, G., Canning, J., Padden, W., Martelli, C., Dligatch, S.: Large area optical mapping of surface contact angle. Opt. Express 25(18), 21127-21144 (2017)

3. Luo, D., Qian, L., Dong, L., Shao, P., Yue, Z., Wang, J., Shi, B., Wu, S., Qin, Y.: Simultaneous measurement of liquid surface tension and contact angle by light reflection. Opt. Express 27(12), 16703-16712 (2019)

4. Horn, B.K.P., Sjoberg, R.W.: Calculating the reflectance map. Appl. Opt. 18(11), 1770-1779 (1979)

5. Woodham, R.J.: Gradient and curvature from the photometricstereo method, including local confidence estimation. J. Opt. Soc. Am. A 11(11), 3050-3068 (1994)

6. Ohno, H.: One-shot three-dimensional measurement method with the color mapping of light direction. OSA Continuum 4(3), 840-848 (2021)

7. Ohno, H.: One-shot color mapping imaging system of light direction extracted from a surface BRDF. OSA Continuum 3(12), 3343-3350 (2020)

8. Ohno, H., Kano, H.: Depth reconstruction with coaxial multiwavelength aperture telecentric optical system. Opt. Express 26(20), 25880-25891 (2018)
9. Ohno, H., Kano, H.: Optical test apparatus and optical test method. US patent 2019/0364267 A1 (2019)

10. ZYGO Corporation. NewView7200. https://www.zygo.com/

11. Howes, W.L.: Rainbow schlieren and its applications. Appl. Opt. 23(14), 2449-2460 (1984)

12. Agrawal, A.K., Butuk, N.K., Gollahalli, S.R., Griffin, D.: Threedimensional rainbow schlieren tomography of a temperature field in gas flows. Appl. Opt. 37(3), 479-485 (1998)

13. Ohno, H., Toya, K.: Reconstruction method of axisymmetric refractive index fields with background-oriented schlieren. Appl. Opt. 57(30), 9062-9069 (2018)

14. Ohno, H., Toya, K.: Scalar potential reconstruction method of axisymmetric 3D refractive index fields with background-oriented schlieren. Opt. Express 27(5), 5990-6002 (2019)

15. Ohno, H., Toya, K.: Localized gradient-index field reconstruction using background-oriented schlieren. Appl. Opt. 58(28), 7795-7803 (2019)

16. Ohno, H., Usui, T.: Gradient-index dark hole based on conformal mapping with etendue conservation. Opt. Express 27(13), 18493-18507 (2019)

Publisher's Note Springer Nature remains neutral with regard to jurisdictional claims in published maps and institutional affiliations. 